\title{
MEMORYLESS SEARCH ALGORITHMS IN A NETWORK WITH FAULTY ADVICE
}

\author{
Nicolas Hanusse* \\ LaBRI - CNRS - Université Bordeaux I, 351 Cours de la Liberation, 33405 Talence, \\ France. \\ hanusse@labri.fr
}

Dimitris Kavvadias
Department of Mathematics, University of Patras, Rio, Greece.
kavadias@ceid.upatras.gr

Evangelos Kranakis ${ }^{\dagger}$

School of Computer Science, Carleton University, Ottawa, ON, K1S 5B6, Canada. kranakis@scs.carleton.ca

\author{
Danny Krizanc ${ }^{\ddagger}$ \\ Department of Mathematics, Wesleyan University, Middletown CT 06459, USA. \\ dkrizancecaucus.cs.wesleyan.edu
}

\begin{abstract}
In this paper, we present a randomized algorithm for a mobile agent to search for an item $t$ stored at a node of a network, without prior knowledge of its exact location. Each node of the network has a database that will answer queries of the form "how do I get to $t$ ?" by responding with the first edge on a shortest path to $t$. It may happen that some nodes, called liars, give bad advice. We investigate a simple memoryless algorithm which follows the advice with some fixed probability $q>1 / 2$ and otherwise chooses a random edge. If the degree of each node and number of liars $k$ are bounded, we show that the expected number of edges to follow in order to reach $t$ is bounded from above by $O\left(d+r^{k}\right)$,
\end{abstract}

\footnotetext{
"Research supported in part by CNRS-Jemstic "Mobicoop" grant.

t Research supported in part by NSERC (Natural Sciences and Engineering Research Council of Canada) and MITACS (Mathematics of Information Technology and Complex Systems) grants.

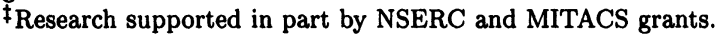


where $d$ is the distance between the initial and target node and $r=\frac{q}{1-q}$. We also show that this expected number of steps can be significantly improved for particular topologies such as the complete graph, the torus, and the spider graph.

Keywords: Data Structure and Algorithms, Distributed Computing, Randomized Algorithms, Graph , Random Walks, Faulty Networks

\section{Introduction}

Searching for a piece of information is one of the most common tasks in a distributed environment. The evolution of the World Wide Web, for example, has led to the development and the wide use of specialized sites called search engines, that are able to provide the user with information on almost any possible query. Therefore, seeking a piece of information in a distributed environment is an algorithmic problem of major significance.

The usual mathematical model of such an environment is a graph (directed or undirected) whose nodes are computers that perform routing of message packets and/or store information. This information can be retrieved by an interrogating program that can be seen as an agent (mobile agent) that visits the nodes of the graph in a certain way moving from node to node using the edges. The purpose of the mobile agent is to locate a specific piece of information or token that resides in a certain node [KK99, KKKS00].

Graph searching is a central procedure in many computational problems. There are numerous techniques and algorithms for this problem that vary from purely deterministic (e.g., depth or breadth-first search) to purely randomized, (e.g. random walks, $\left.\left[\mathrm{AKL}^{+} 79, \mathrm{KR} 95\right]\right)$. A variety of versions of the problem have been proposed that capture the requirements of different problem settings. For example, the agent may have limited knowledge of the topology of the network (e.g., searching in an unknown graph $\left[\mathrm{MHG}^{+} 88\right]$ ) or it may exploit some geometric properties of the network (e.g., searching in the plane [BYCR93]). The efficiency of the search algorithm that the agent executes is measured, as usual, by the time it takes to find the token and/or its memory requirements.

In this paper we study the efficiency of searching algorithms where the advice is based on shortest path information that an agent receives when it arrives at a node. That is, the computer that resides at the node advises the agent by revealing to it an edge that is the beginning of a shortest path from the current node to the token. This kind of advice is common in the IP protocol where routing is done usually through the shortest available path. However, as is common in practice, we also assume that certain nodes-called liars-may give faulty information. This may model a situation where a computer is malfunctioning or its knowledge about the topology of the graph and/or the position of the token, is outdated. The searching problem in the presence of liars has been proposed and studied in [HKK00]. In that paper, several deterministic algorithms were proposed for various network topologies. In the present paper we propose a randomized algorithm for the same problem focusing mainly on 
the memory requirements. The reason for this is simple: the size of a network such as the Web is such that any agent that accumulates information in its traversal of the network can end up having devastating memory requirements. We are therefore interested in memoryless algorithms. It is easily seen however, that any memoryless algorithm that only receives shortest path information has to use randomization in order to avoid deadlocks. For specific topologies, our randomized algorithm is more competitive than any deterministic algorithm. For example, for the worst-case distribution of $k$ liars, to find deterministically a token in the complete graph of $n$ nodes, we need at least $O(k)$ steps and $O(\log k)$ bits of memory. Using our memoryless randomized algorithm, on average $O(1)$ steps are sufficient.

\subsection{Definitions}

A network is for our purposes an undirected graph $G=(V, E)$ where $V$ is the set of nodes and $E$ the set of edges. There are two specific nodes in the graph, the start node which we denote by $s$ and the token which we denote by $t$. All nodes point to an edge incident to them. Each node is either a truth-teller or a liar. A node of the former kind points to an edge which is the start of a shortest path from that node to the token. A node of the latter kind may point to any incident edge except the start of a shortest path.

In our model, once a node is characterized it always gives the same response throughout the execution of the algorithm. Before the execution of the algorithm, all nodes are truth-tellers. An adversary then selects $k$ nodes out of the $n$ and substitutes their advice with bad advice. We will be working mainly in the strong adversary model in which the adversary can also modify the advice of each truthteller as long as they continue to provide an edge on a shortest path. We show in Section 4.2 a spectacular difference between a weak and a strong adversary model whenever there are many paths to go to the destination. Intuitively, a strong adversary can break any coordination of truth-tellers that might be of help to the agent. The agent is unaware of the kind of node in which it arrives.

Parameters that will be used are the total number of liars $k$ and the initial distance $d$ between $s$ and $t$. We shall also be interested in the topology of the graph and in its diameter which we denote by $D$.

\subsection{Markov Chains and zero memory algorithms}

We use the terminology of [Nor97] of Markov Chains and random walks. Let $P=\left(0 \leq p_{x, y} \leq 1 \mid x, y \in V\right)$ be a stochastic matrix, i.e. the sum of every row is equal to 1. A discrete-time Markov Chain on a finite set of states $V$ is a sequence of random variables $V_{0}, V_{1}, \ldots$ where $V_{i} \in V$ and such that $V_{i+1}$ depends only on $V_{i}$ and $\operatorname{Pr}\left(V_{i+1}=x \mid V_{i}=y\right)=p_{x, y}$. The matrix $P$ is called the transition probability matrix. In the context of a biased random walk in a graph, $p_{x, y}$ denotes the probability to go from node $x$ to node $y$ in one step. 
A node $x$ leads to a node $y$ if $\operatorname{Pr}\left(V_{j}=y\right.$ for some $\left.j \geq i \mid V_{i}=x\right)>0$. A state $y$ is absorbing if $y$ leads to no other state. The expected hitting time or hitting time $\mathbb{E}_{x}^{y}$ is the expected or mean number of steps starting from node $x$ to reach node $y$. In our paper, the token $t$ is the only absorbing state and the expected number of steps to reach $t$ from $s$ is noted $\mathbb{E}_{s}^{t}$.

We will make use of the following well-known theorem for Markov chains:

Theorem 1 The vector of hitting times $\mathbb{E}^{t}=\left(\mathbb{E}_{x}^{t}: x \in V\right)$ is the minimal non-negative solution to the system of linear equations:

$$
\left\{\begin{array}{l}
\mathbb{E}_{t}^{t}=0 \\
\mathbb{E}_{x}^{t}=1+\sum_{y \neq t} p_{x y} \mathbb{E}_{y}^{t} \text { for } x \in V
\end{array}\right.
$$

\subsection{Description of the Algorithm}

In the model above the mobile agent executes a simple, memoryless algorithm whose goal is to take advantage of the advice that it gets in a node but at the same time avoid deadlocks that may arise in some cases. The algorithm SEARCH is as follows:

1 The agent arrives at a node of degree, say, $\Delta$ and if it discovers the token it halts. Otherwise, it asks the node for advice.

2 The node responds by pointing to one of the edges incident to it.

3 The agent then flips a biased coin and with probability $q$ it follows the advice. That is, it moves to the adjacent node which is the other endpoint of the edge. If it decides not to follow the advice (an event of probability $1-q$ ), it selects uniformly another edge among the remaining $\Delta-1$ incident edges.

4 It then moves to the other endpoint of the selected edge.

5 The above steps are repeated at the new node.

The idea behind this algorithm is that the agent expects, as a general rule, the advice to be correct. That is, the agent assumes that the majority of the nodes are truth tellers and that following their advice will bring the agent faster to the token. Therefore the probability $q$ is taken to be more than $1 / 2$. The agent however cannot trust completely the advice that it receives as this may lead to a deadlock. Consider for example a case where the endpoints of an edge are nodes pointing to each other. It is clear that at least one of the nodes is a liar and that if the agent chooses to always follow the advice it will move back-and-forth between these two nodes forever. By allowing the agent not to trust the advice with some positive probability, we expect that, eventually, it will be able to get out of situations like this.

\footnotetext{
${ }^{1}$ In Norris' book [Nor97], the hitting time is the minimum amount of time needed to reach a node.
} 
We note that the actions of the algorithm SEARCH resembles that of a biased random walk as studied by Azar et al. [ $\left.\mathrm{ABK}^{+} 96\right]$. In that case however, on each step a coin is flipped which determines whether the current node a liar or not. Thus the number of liars is a random variable which is a function of the bias, and a node can in one instance be a liar on the next visit of the agent, a truthteller. In our case, the number and positions of the liars remains fixed throughout the execution of the algorithm. The analysis of both cases relies on Markov chains.

\subsection{Results and structure of the paper}

Our main task is to analyze the probabilistic algorithm SEARCH presented in Section 1.3. Our algorithm is memoryless and we are interested in the time complexity to reach the destination for the worst distribution of liars. We count each edge used by the mobile agent as a single step. We assume the majority of the nodes are truth tellers. As a consequence, we consider that $q>1 / 2$.

In Section 2, we consider the chain graph where we show the expected number of steps of our algorithm is $O\left(d+r^{k}\right)$, for $r=\frac{q}{1-q}$. In Section 3 we show that we can generalize this result to an arbitrary graph. We use this to show that if the mobile agent has approximate knowledge of the distance and the number of liars, the token can be reached on average in $d+O\left(d k / \ln \left(d k^{-6}\right)\right)$ steps.

In Section 4, we consider specific topologies. In Section 4.1, we deal with the complete graph for which we prove that, even if the number of liars is large, the expected number of steps to reach the destination is a constant. However, in the complete graph, the distance is the constant 1 . In the torus, studied in Section 4.2 , we prove a lower bound of $\Omega\left(d+r^{k}\right)$ steps in the strong adversary model. For the same distribution of liars, the expected number of steps is $O(d)$ in the weak adversary model. In Section 4.3 we study the case of having different shortest paths to reach the token. For spider graphs of $N$ nodes per layer, the algorithm leads to an expected number of steps of $O\left(d+N^{2}+r^{O(k / N)}\right)$ using the fact that the mobile agent has the possibility to avoid obstacles, i.e. liars, to reach an area with only truth-tellers.

Due to space limitations, the proofs are not given.

\section{Chain}

It turns out that a very simple graph, namely the chain - or line - behaves as bad as any graph with the same number of liars. This is of no surprise as in a line all nodes -and therefore all liars- have to be visited as there is only one way to reach the token. We begin our study therefore with the chain graph.

Consider the $n$-vertex chain graph $0,1, \ldots, n-1$. Each node $i$ except the first and the last, is only connected to its predecessor $i-1$ and its successor $i+1$. We suppose that $0 \leq s \leq t$. If $i$ is therefore a truth teller, $i<t$, the probability that the agent will move to node $i+1$ is $p_{i, i+1}=q$ (trust the advice) and to move to node $i-1$ is $p_{i, i-1}=1-q$ (not trust the advice). The corresponding 
probabilities for $i$ being a liar are of course $p_{i, i+1}=1-q$ and $p_{i, i-1}=q$. We assume that $p_{0,1}=p_{n-1, n-2}=1$.

Set $r=\frac{q}{1-q}$. Since $q>1 / 2, r>1$. To each edge $i$ between node $i$ and $i+1$, we assign a weight $w_{i+1}$ with $w_{1}=1$ and $w_{i+1}=\frac{p_{i, i+1}}{p_{i, i-1}} w_{i}$. So $w_{i+1}=r w_{i}$ if $i$ is a truth teller and $w_{i+1}=\frac{w_{i}}{r}$ otherwise. For convenience, let $W_{j}=\sum_{i=0}^{j} w_{i}$.

The following result is given in [AF][Chapter 5], and constitutes a version of theorem 1 mentioned above:

Lemma 1 (Essential edge lemma) Let $s<t$ be two nodes of the weighted chain $C=\left(w_{1}, w_{2}, \ldots, w_{n-1}\right)$. For a random walk in $C$,

$$
\mathbb{E}_{s}^{t}=t-s+2 \sum_{j=s+1}^{t} \sum_{i=1}^{j-1} w_{i} w_{j}^{-1}
$$

Our main result for the chain follows from the following two lemmas.

Lemma 2 The worst case distribution of liars before $s$ that maximizes $\mathbb{E}_{s}^{s+1}$ is to place them consecutively and close to $s$.

Lemma 3 Let $l_{i}$ be the number of liars between nodes 0 and $i$. Let $\beta_{i}$ be the number of consecutive truth-tellers between the last liar and $i$. Then, for $l_{i} \geq \beta_{i}$, we have $\mathbb{E}_{i}^{i+1}<1+\left(\frac{6 r^{l_{i}-\beta_{i}+1}}{r-1}\right)$ and for $l_{i}<\beta_{i}, \mathbb{E}_{i}^{i+1}<1+\frac{6}{r-1}$. For $r$ constant, it turns out $\mathbb{E}_{i}^{i+1}=O\left(1+r^{l_{i}-\beta_{i}}\right)$.

Theorem 2 Let $s<t$ be two nodes of the chain with $k$ liars and $q$ be the probability of trusting the advice. Let $r=\frac{q}{1-q}$ and let $d$ be the distance between $s$ and $t$. Then the expected number of steps to reach $t$ from $s$ using algorithm SEARCH is less than $d\left(1+\frac{6}{r-1}\right)+\frac{6 r^{k+3}}{(r-1)^{3}}$. If $q$ is constant this is $O\left(d+r^{k}\right)$.

Theorem 3 For $r$ constant and for the worst distribution of $k$ liars in the line, $\mathbb{E}_{s}^{t}=\Omega\left(d+r^{k}\right)$.

\section{Arbitrary Graph}

In this section we study the expected number of steps to reach the token in an arbitrary graph. Let $\mathrm{G}$ be any graph. Starting from $t$, arrange all nodes of $G$ in layers according to their distance from $t$. Denote by $L_{i}, i=0, \ldots, m$ the set of nodes that are at distance $i$ from $t$. Here, $m$ is the maximum distance between $t$ and any other node. Construct the following Markov chain $\left(Q_{j}\right), j=0, \ldots, m$. $Q$ has $m+1$ states each corresponding to a layer of the graph. As for the transition probabilities let the probability of moving from state $Q_{i}$ to state $Q_{j}$, $p_{i, j}$, be:

$$
p_{i, j}=\left\{\begin{aligned}
q & : \text { if } i=j+1 \text { and all nodes in layer } L_{i} \text { are truth-tellers; } \\
\frac{1-q}{\Delta_{i}-1} & : \text { if } i=j+1 \text { and there exists at least one liar in layer } L_{i} \\
1-p_{i, i-1} & : \text { if } i=j-1 \\
0 & : \text { if }|i-j| \neq 1
\end{aligned}\right.
$$


In the above, we denote by $\Delta_{i}$ the maximum degree among all nodes of layer $L_{i}$. In effect, the Markov chain that we defined has one state for each layer of the graph and its transition probability from state (layer) $Q_{i}$ to state $Q_{i-1}$ is the minimum among the probabilities to move from any node in layer $L_{i}$ to a node in layer $L_{i-1}$, that is, one step closer to the token: When the agent is on a layer with truth-tellers only, the probability of moving closer is of course $q$ and moving further is $1-q$. When the agent is on a layer with at least one liar then it may happen that it resides on a node $i$ which is actually a liar, in which case the probability of moving closer is at least $\frac{1-q}{\Delta_{i}-1}$. The initial state of $(Q)$ is of course the state that corresponds to the layer which includes the starting node $s$. This is state $Q_{d}$ since we have assumed that the token is at distance $d$ from our initial position.

By our choice of transition probabilities the event of moving closer to state $Q_{0}$ from any state of $Q$, is less probable than moving from any node of $G$ to another node closer to $t$. It is therefore clear that the expected number of steps to reach node $t$ when starting from $s$, is less or equal than the expected number of steps to reach state $Q_{0}$ when starting from state $Q_{d}$.

The so constructed Markov chain $(Q)$ is a line in which the distance between the initial and target nodes is $d$ and with at most $k$ liars. Notice that while the truth-tellers in $(Q)$ preserve their usual probabilities (i.e. $q$ to go closer and $1-q$ to go furher), the liars are "stronger" in the sense that the probability of going closer is now only $p_{i, i-1}=\frac{1-q}{\Delta_{i}-1}$. (Notice the change in the numbering of nodes in the current chain $(Q)$.) Let $\Delta=\max _{i} \Delta_{i}$. Going back to the proof of Theorem 2 of the previous section, we see that the effect of a liar in the application of Lemma 1 is to divide the weight of the next edge by $r$. In our case $r=\frac{p_{i, i+1}}{p_{i, i-1}}=\frac{\Delta_{i}-2+q}{1-q}$. In the case of the current chain $(Q)$ the parameter $r$ is always less than $\frac{\Delta-1}{1-q}$. The above observation along with Theorem 2 therefore gives:

Theorem 4 Let $G$ be any network of maximal degree $\Delta$ with $k$ liars in which the distance between the initial node $s$ and the token $t$ is $d$. Then the expected number of steps of a mobile agent to reach $t$ is less than $d\left(1+\frac{6}{r-1}\right)+\frac{6 r^{k+3}}{(r-1)^{3}}$ where $r=\frac{\Delta-1}{1-q}$.

\subsection{Tuning the mobile agent}

As algorithm SEARCH is memoryless, the only parameter that can be adjusted to improve performance is the amount of "belief" of the agent to the advice it receives, that is, probability $q$. Even so, there can be many different versions of this problem depending on what we might consider known to the agent. Interesting choices include the distance, the number of liars, the distribution of liars, the location of the initial node in the network, the topology etc. As an easy example, we consider the worst topology, i.e the line, the worst distribution of liars and we assume that the order of the distance and the number of liars are known. 
Theorem 5 Let $G$ be any network with $k$ liars in which the distance between the initial node $s$ and the token $t$ is $d$. For $d>\frac{(k+3)^{6}}{2}$, taking $r=$ $1+\frac{\ln \left(2 d /(k+3)^{6}\right)}{2(k+3)}$, the expected number of steps of a mobile agent to reach $t$ is $d+O\left(d k / \ln \left(d k^{-6}\right)\right)$.

\section{Special Graphs}

The above disapointing bound of $O\left(d+r^{k}\right)$ comes from the fact that there may exist a bottleneck between the initial node and the token where all $k$ liars may reside. If however the topology of the graph allows multiple paths, then things can become much better as for example in the complete graph. Moreover multiple paths give rise to new interesting problems as a truth teller may now have a choice of shortest paths to point to. We show by giving a specific example in the torus that different advice from the truth tellers do make a difference in the expected number of steps.

\subsection{Complete Graph}

For the complete graph, we prove that, even when the number of liars is large, the expected number of steps to reach the destination is a constant.

Theorem 6 Assume that in $K_{n}$ (the complete graph with $n$ nodes) the number of liars is $k=c n$, where $c$ is a constant, $0<c<1$. Then the expected number of steps to reach the token is $\frac{1}{(q-q c)(1-q)}+O\left(\frac{1}{n}\right)$.

Corollary 1 In the complete network $K_{n}$ containing $\Theta(n)$ truth-tellers, the expected number of steps to reach the destination is $O(1)$.

\subsection{Multiple paths. The case of the torus}

The effect of liars on the required time to reach the token may sometimes be affected by the advice of the truth tellers. This can happen in cases where multiple shortest paths exist from some nodes to the token and consequently multiple possibilities exist for the advice that the agent receives from a truth teller. The purpose of this section is to demonstrate this by studying the running time of the algorithm for a specific setup on the torus. A torus of $n=n_{1} n_{2}$ nodes is a the graph obtained by the cartesian product of two cycles of length $n_{1}$ and $n_{2}$. For convenience, we label each node $u=(i, j)$ with $i=\left[-\left\lceil n_{1} / 2\right\rceil,\left\lfloor n_{1} / 2\right\rfloor\right]$ and $j=\left[-\left\lceil n_{2} / 2\right\rceil,\left\lfloor n_{2} / 2\right\rfloor\right]$. A node $v=\left(i^{\prime}, j^{\prime}\right)$ is a neighbor of $u$ if $\left|i-i^{\prime}\right|=1\left(n_{1}\right)$ and $\left|j-j^{\prime}\right|=1\left(n_{2}\right)$. The torus is an appropriate example as it is a common network architecture with symmetry that allows relatively easy calculations.

Consider the following situation: Graph $\mathrm{G}$ is a torus with diameter $D$ much larger than the distance $d$ between the starting node and the token. The token is placed at the origin $(0,0)$ while the $k$ liars occupy the nodes from $(0,1)$ to $(0, k)$. The starting node $s$ is the node $(0, d)$. We distinguish two cases: 
Case 1 All truth tellers with coordinates $(x, y)$ point left if $x>0$ and right if $x<0$. They point to the appropriate (unique) direction if they lie on the $y$ axis.

Comments: This case is a lower bound for this kind of adversary.

Case 2 All truth tellers with $y \neq 0$ point either up or down depending on their position. That is, all truth tellers with $y>0$ point down and all truth tellers with $y<0$ point up. They point to the appropriate direction if they lie on the $x$ axis.

We next study the expected number of steps to reach the token in each of the above cases. We have the following result:

Theorem 7 The expected number of steps to reach the token in Case 1 is $\Omega\left(d+r^{\prime k}\right)$ where $r^{\prime}$ is a constant depending on $q$. In contrast, in the second case the expected number of steps is proportional to $d$.

\subsection{Spider Graph}

We now turn our attention to a particular graph which reveals some additional merits of our searching algorithm. We call this graph spider graph because it resembles a spider's web. A node $u$ of the spider graph has the polar coordinates $(x, y) \in\{1, . ., n / N\} \times\{0, \ldots, N-1\} \cup\{(0,0)\}$ where $x$ represents the radius, i.e. the distance to the token and $y$ the angle. Node $u \neq(0,0)$ has four neighbors $(x, y+1),(x, y-1),(x-1, y)$ and $(x+1, y)$. The node $(0,0)$ is called the center of the spider graph. We assume that the token lies on the center node and is connected to $N$ nodes $(1,0), \ldots,(1, N-1) . N$ is called the density which is the number of nodes on a cycle of the graph. Less important parameter is the number of cycles, or equivalently the number of nodes on any radius of the graph. This is because we assume, as usual, that the majority of the nodes are truth tellers and consequently far enough from the center all nodes are truth tellers. We are therefore interested in the part of the spider graph around the center and at a radius approximately the maximum distance between $d$ (the distance of the initial node) and the distance of the furthest liar.

All truth tellers point of course at the center of the graph while we assume for simplicity that all liars point at the opposite direction. Hence with probability $p_{r}=q+(1-q) / 3$ the agent will move along its current radius and with probability $p_{c}=1-p_{r}=2(1-q) / 3$ it will change radius and move to a neighboring node on the current cycle. Since the probabilities to move on a cycle clockwise and counterclockwise are equal, if we ignore the moves that the agent does along a radius, its resulting motion is an unbiased random walk on a ring with $N$ nodes. This last observation explains how the agent is able to avoid areas with high concentration of liars: It "probes" the area in front (toward the token) in an attempt to get closer to the token. We know from Section 2 that if there are $k$ liars in front, then it will take the agent $O\left(r^{k}\right)$ steps to pass them. But since the agent also moves around on the current ring 
continuously trying to get closer to the token, it will eventually discover areas with no liars or with only a few liars and it will pass from there.

We label the set of radii from 0 to $N-1$ in the clockwise direction. We denote by $\left(X_{i}, Y_{i}\right)$ the polar coordinates of the mobile agent at time $i$ where $X_{i}$ corresponds to the distance from the center and $Y_{i}$ is the label of the current radius. By convention, $\left(X_{0}, Y_{0}\right)=(d, 0)$. The following result comes from the mixing time in a ring of $N$ nodes [AF]:

Lemma 4 If $q$ is constant then for $i=\Omega\left(N^{2}\right)$ and for all $y \in[0, N-1]$, $\left|\operatorname{Pr}\left(Y_{i}=y\right)-1 / N\right|=\epsilon$.

Let $t_{x}$ be the number of truth-tellers of layer $x$ (at distance $x$ from the destination). Let $p_{x}^{i}$ be the probability, knowing that $X_{i}=x$, to go from layer $x$ to layer $x-1$ at time $i$.

Lemma 5 After $i=\Omega\left(N^{2}\right)$ steps, $p_{x}^{i}>1 / 2$ if $t_{x}>c N$ with $c=\frac{1+2 q}{2(4 q-1)}$.

The next result shows that if the density of the spider graph is significant, the time to reach the token can become much better.

Theorem 8 In a spider graph of density $N$, if $q$ is constant, the expected number of steps to reach the token is upper bounded by $O\left(d+N^{2}+r^{\left\lfloor\frac{2 k(4 q-1)}{(6 q-3) N}\right\rfloor}\right)$.

\section{Conclusion}

We have presented "memoryless" randomized algorithms to search for an item $t$ contained in a node of a network, without prior knowledge of its exact location and under the assumption that some nodes, called liars, may give bad advice. We have provided an algorithm and studied its performance in an arbitrary network and also considered different topologies like the complete graph, the torus, and the spider graph. It would be interesting to consider techniques similar to [KR95, BKRU89] and [MR95][Chapter 6] in order to study time-memory tradeoffs, as well as search algorithms for multiple mobile agent systems in our model.

\section{References}

[ABK $\left.{ }^{+} 96\right]$ Y. Azar, A. Broder, A. Karlin, N. Linial, and S. Phillips. Biased random walks. Combinatorica, 16:1-18, 1996.

[AF] D.J. Aldous and J.A. Fill. Reversible markov chains and random walks on graphs. (book in preparation, available on the web from http://www.stat.berkeley.edu/users/aldous/book.html).

[AKL $\left.{ }^{+} 79\right] \quad$ R. Aleliunas, R. M. Karp, R. J. Lipton, L. Lovász, and C. Rackoff. Random walks, universal traversal sequences and the complexity of maze problems. In Proc. 20th FOCS, pages 218-223, 1979.

[BKRU89] A. Broder, A. K. Karlin, P. Raghavan, and E. Upfal. Trading space for time in undirected $s-t$ connedtivity. In Proc. STOC, pages 543-549, 1989. 
[BYCR93] R. Baeza-Yates, J. Culberson, and G. Rawlins. Searching in the plane. Information and Computation, 1993.

[HKK00] N. Hanusse, E. Kranakis, and K. Krizanc. Searching with mobile agents in networks with liars. In Proc. EUROPAR'2000, LNCS 1900, pages 583-590, Munich, 2000.

[KK99] E. Kranakis and D. Krizanc. Searching with uncertainty. In Proc. SIROCCO'99, Carleton Scientific, pages 194-203, 1999.

[KKKS00] L. M. Kirousis, E. Kranakis, D. Krizanc, and Y. Stamatiou. Locating information with uncertainty in fully interconnected networks. In Proc. DISC'2000, LNCS 1914, pages 283-296, Toledo, Spain, 2000.

[KR95] A. R. Karlin and P. Raghavan. Random walks and undirected graph connectivity: A survey. In Discrete Probability and Algorithms, Institute of Mathematics and its Applications, Vol., 72, Springer Verlag, 1995, pages 95-101, 1995.

[MHG $\left.{ }^{+} 88\right]$ N. Megiddo, S. Hakimi, M. Garey, D. Johnson, and C. Papadimitriou. The complexity of searching a graph. Journal of the ACM, 1988.

[MR95] R. Motwani and P. Raghavan. Randomized Algorithms. Cambridge University Press, 1995.

[Nor97] J.R. Norris. Markov Chains. Cambridge University Press, 1997. 